GPPS-TC-2019-0087

\title{
REAL GAS EFFECTS ON THE OFF-DESIGN PERFORMANCE OF A SCO2 COMPRESSOR
}

\author{
Robert Pelton \\ Hanwha Power Systems Americas \\ rob.pelton@hanwha.com \\ Houston, TX, USA
}

\author{
Sewoong Jung \\ Hanwha Power Systems Americas \\ sewoong.jung@hanwha.com \\ Houston, TX, USA
}

\section{ABSTRACT}

Off-design analysis of turbomachinery driven systems are often performed based on non-dimensional component performance maps. According to aerodynamic similarity principles these maps are assumed to remain constant over a broad range of operating conditions. While it is understood that the scaling laws only strictly apply to ideal gasses, constant performance maps are often used in evaluating real gas cases because a simple method to estimate the real gas effects is not available. In the case of sCO2 Brayton cycles, there are performance advantages to operating the compressor near the critical point of the working fluid. The properties of $\mathrm{CO} 2$ near the critical point exhibit pronounced real gas characteristics where small changes in temperature or pressure correspond to wide variations in the density, speed of sound and $C p$. To assess the effects of real gas properties on the compressor performance a careful CFD study was conducted to explore the variation in nondimensional performance associated with small changes in inlet temperature and pressure that would be expected in typical off-design operation of an air cooled sCO2 Brayton cycle. The results of the analysis find that two key map characteristics change significantly with inlet conditions, the head coefficient and the choke margin. These changes in compressor performance directly affect the cycle power balance, the performance of the other components in the loop, and the cycle transients.

A review of the analytic performance models suggest that the variation in choke margin is proportional to the change in inlet sonic velocity. Since sonic velocity can be assessed easily, the non-dimensional performance maps can be adjusted to match the gas properties to more accurately capture the expected stable operating range of a given geometry. The results highlight the need to carefully select the compressor inlet design point of the cycles to avoid operational limitations. If the design point is selected too close to the critical point, then at certain off-design conditions the stage may choke and be unable to pass the required flow.

\section{INTRODUCTION}

The overall efficiency of a supercritical carbon dioxide (sCO2) Brayton cycles is maximized as the compressor inlet conditions approach the critical point (Clementoni and Cox, 2018). While operation near the critical point causes some concern due to variability of the gas properties, the cycle performance advantages of operating in this region make it necessary. Figure 1 below shows the cycle efficiency advantage of operating near the critical point for a simple recuperated Brayton cycle with a turbine inlet temperature near $500^{\circ} \mathrm{C}$ and a max cycle pressure of 230barA. The overall cycle performance is increased as the compressor inlet temperature decreases. However, in cases where the cycle is air cooled, the compressor inlet temperature may vary significantly due to the changing ambient conditions so understanding the performance at off-design conditions is critical.

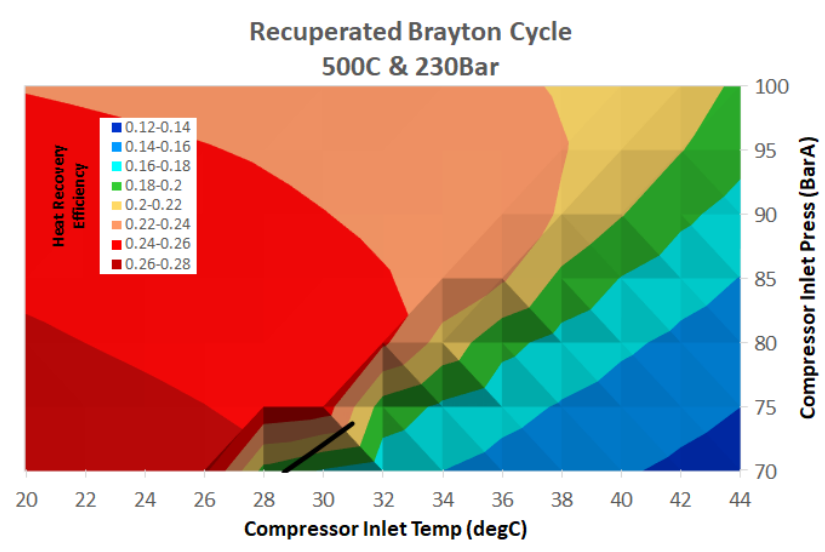

Figure 1: sCO2 Brayton Cycle Modelling Showing the Advantages of Operating Near the Critical Point 
According to aerodynamic similarity principles (Buckingham, 1914) several key non-dimensional parameters are shown to remain constant over a broad range of operating conditions. (Japikse, 1996). To calculate performance when the inlet conditions change, the performance of the stage can be accurately calculated based on the non-dimensional map of the design condition performance (ASME, 1997). Typically, off-design component performance of turbomachinery is calculated based on the non-dimensional parameters including head coefficient, $\Psi$, flow coefficient, $\phi$, and isentropic efficiency, $\eta$, Mach number and Reynolds number (Dufour, et al., 2006). To model basic cycle performance it is adequate to have maps of head coefficient and efficiency vs. flow coefficient. This allows the pressure ratio and power of the compressor to be estimated for a range of different inlet conditions.

To accurately evaluate the operation of the cycle at a range off-design operating conditions it is critical to have accurate representation of the performance of the compressor. While it is understood that the scaling laws only strictly apply to ideal gasses, constant performance maps are often used in evaluating real gas cases because a simple method to estimate the real gas effects is not available. (Bennett, et al., 2017) For a sCO2 Brayton cycle the main compressor inlet conditions will be near the critical point and subject to the most significant variation in fluid properties.

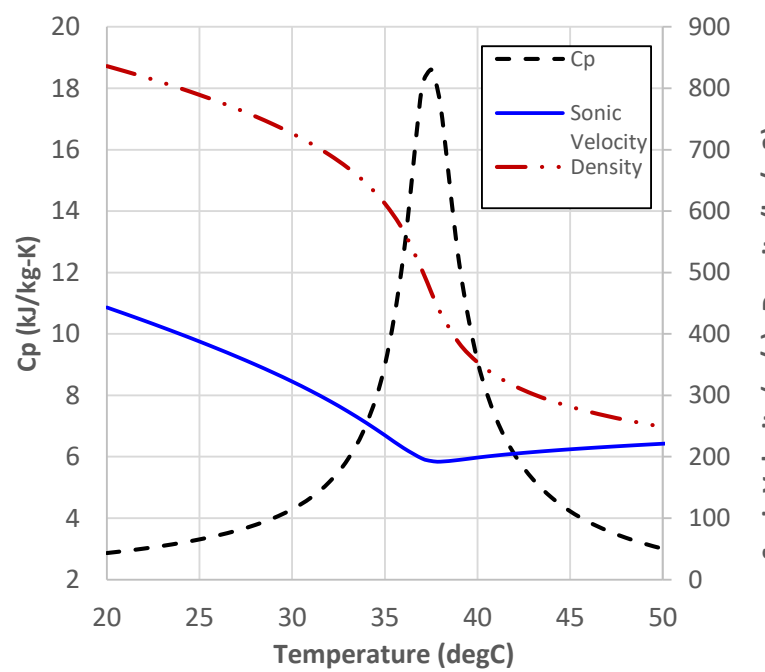

Figure 2: Fluid Properties of $\mathrm{CO} 2$ at $85 \mathrm{barA}$

The properties of $\mathrm{CO} 2$ near the critical point exhibit pronounced real gas characteristics where small changes in temperature or pressure correspond to wide variations in the density, speed of sound and specific heat, $C p$ (Angelino, 1969, Fomin, et al, 2014, Holste, 2013, Li, 2008). Figure 2 shows the variation in $\mathrm{Cp}$, sonic velocity and density of $\mathrm{CO} 2$ at 85 barA across a range of temperatures from 20 to $50^{\circ} \mathrm{C}$. Below the critical temperature, $31^{\circ} \mathrm{C}$, the density of the fluid is high and $\mathrm{CO} 2$ behaves more like a liquid. With an increase in temperature of just $10^{\circ} \mathrm{C}$ the fluid density is reduced by more than half and the fluid characteristics are more similar to a gas. The sonic velocity is also shown to have a minimum near the critical point and rising on either side of the critical temperature. Also, the specific heat of the gas, $C p$, rises dramatically near the critical point. The design of the compressor must accommodate these potentially rapid fluctuations and the cycle modelling must account for the performance of the compressor at varying inlet conditions.

\section{METHODOLOGY}

The compressor geometry evaluated for this study was a design developed specifically for a $\mathrm{sCO} 2$ recompression Brayton cycle. The impeller has a flow coefficient 0.064, with a diameter of $116 \mathrm{~mm}$ and an isentropic head coefficient of 0.5. The design point of the compressor used for sizing was $37^{\circ} \mathrm{C}$ and 85.1 barA. The basic geometry was based on the impeller design given in (Pelton and Jung, 2018) and (Li, et al., 2018). The impeller geometry was simplified for this study and was defined as a fully covered impeller. Although this study focused on a compressor operating in supercritical $\mathrm{CO} 2$, the results are expected to be applicable to any centrifugal compressor operating near the critical point of a fluid. Compressor performance curves were calculated using computation fluid dynamics (CFD) for a range of inlet conditions. The P-h diagram, in Figure 3, shows the inlet conditions evaluated. The temperature was varied from $20^{\circ} \mathrm{C}$ to $50^{\circ} \mathrm{C}$, representative of a range of temperatures that may exist when air cooling is used. A range of inlet pressures between 80 and 90barA were evaluated since many sCO2 Brayton cycles also consider varying the low side pressure to maximize the cycle output at off-design conditions (Wilkes, et al., 2016). All of the inlets conditions were evaluated at pressures above the critical pressure.

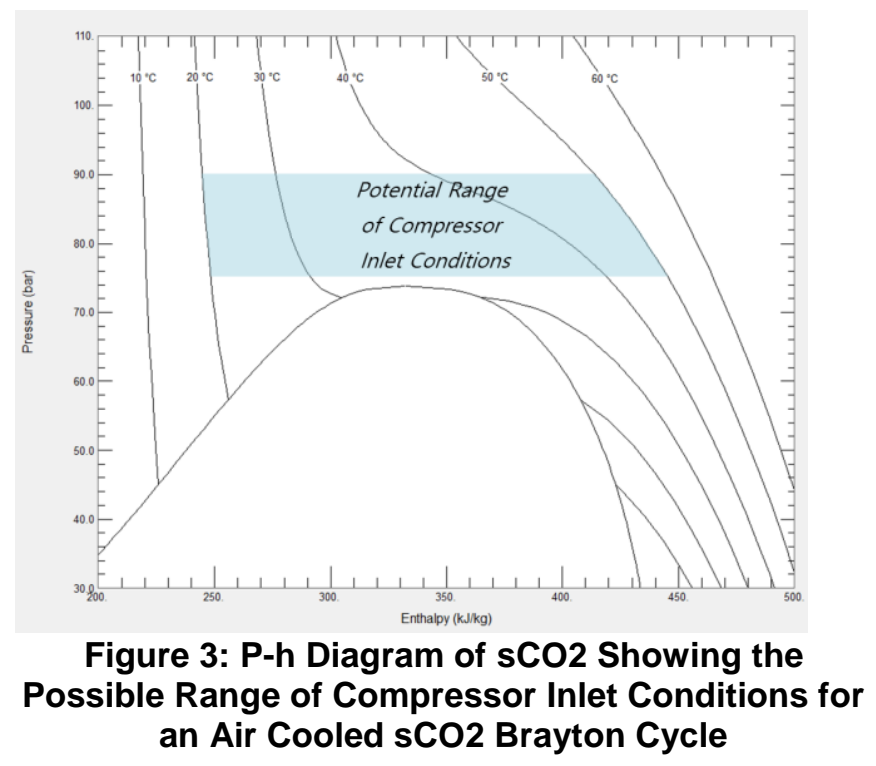

Figure 4 shows the computational domain and mesh of the CFD analysis, including the axial inlet, impeller, and a vaneless diffuser. A volute is expected as the exit element, but was not included in this analysis. Since a vaneless diffuser was applied to this stage, the entire domain was assumed to be a rotating and eliminated any non-matching interfaces. However, actual rotating and stationary passage areas were defined at the boundary conditions. Compressor performance maps were generated using CFD for a range of inlet conditions. The commercial CFD program, NUMECA Fine/Turbo was used for numerical computations applying 
the Spalart-Allmaras one-equation turbulence model with wall functions. The total structured hexahedral mesh size of 1 million cells was constructed via NUMECA's Autogrid 5. An average y+ value of less than 200 was used and wall functions were applied to allow a coarse mesh sufficient to resolve the boundary layer and complete the CFD analysis in a reasonable amount of time. Using wall functions was deemed acceptable since this study was focused on capturing general performance trends associated with the variability in the bulk fluid properties at nominal operating conditions. The additional detail in the flow structure that could be resolved by modelling the full boundary layer are assumed to have a minimal affect the overall stage performance trends.

The total pressure and temperature with a given velocity direction were defined for inlet boundary conditions while a fixed averaged static pressure was applied at the outlet boundary conditions. At low flow near stall, the mass flow rate was specified instead of the static pressure as the outlet boundary condition to improve convergence. The inlet turbulent viscosity was defined by ratio of the turbulent viscosity to the bulk fluid viscosity. A viscosity ratio of 5 was specified to be compatible with the Spalart-Allmaras turbulence model. In addition, non-slip and adiabatic wall boundary conditions were applied. The solution was determined to be converged when the residual value of the root-mean-square (RMS) error was less than $10^{-6}$. Real gas properties were used based on NIST REFPROP (Lemmon, 2013) in this CFD analysis.

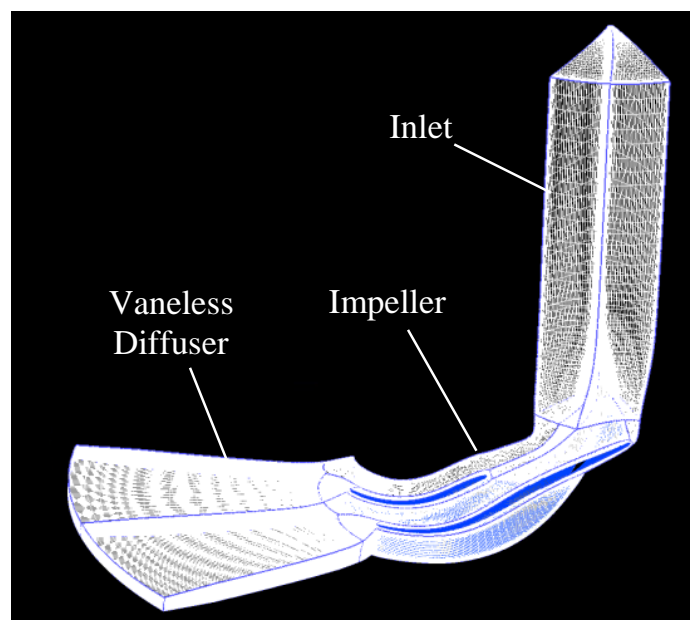

Figure 4: Single Passage Computational Domain and Mesh

A grid sensitivity study was conducted to determine the optimal grid resolution for economical computation runs. Five different mesh sizes varied from 0.46 million to 1.6 million grid points were tested for this study. Figure 5 shows the impact of the performance due to the different grid resolutions at the design condition. It was found that resulting difference was less than $0.5 \%$ for the isentropic efficiency across the range of grid refinements. However, there was a more noticeable change in head at coarse grids, 0.64 million and 0.46 million nodes. Therefore, a total of 1 million grids points was selected for the current work considering computational cost to run the large number of simulations needed for the comparison of the different inlet conditions.

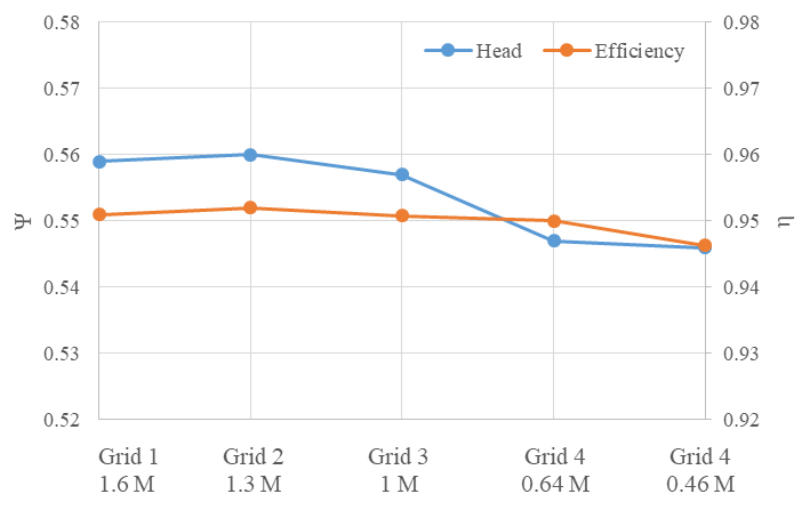

\section{Figure 5: Comparison of Head Coefficient and Isentropic Efficiency at 5 Different Mesh Size}

In this study, the minimum stable operation flow point was identified as the flow rate when the static pressure does not increase with a reduction in mass flow rate, or a stable numerical solution could not be achieved. Choke flow was defined as the flow rate when the mass flow rate no longer increased with a reduction in the discharge static pressure.

\section{RESULTS AND DISCUSSION}

The compressor performance maps are shown below for three different inlet pressures of 80,85 and 90barA. Unlike for an ideal gas, the head coefficient and flow coefficient curves change significantly as the inlet conditions are varied. The head/flow maps for this compressor show two distinct changes from the design condition map as the inlet temperature is varied. First, the choke flow rate varies significantly with temperature. Second, the magnitude of the head coefficient changes, although the slope of the curve remains fairly constant. Similar trends are evident at each of the three different inlet pressures evaluated. The variations become more extreme as the inlet pressure approaches the critical pressure.

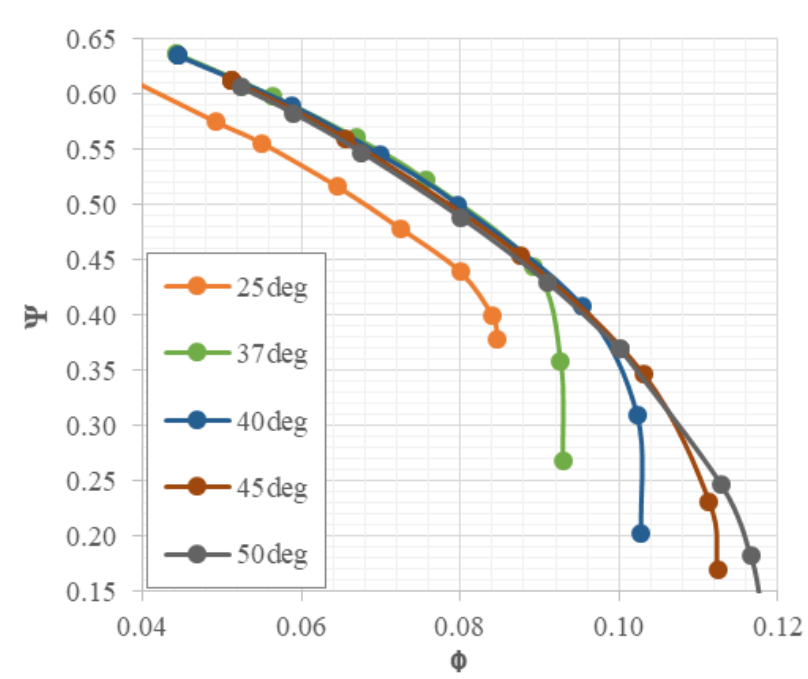

Figure 6: Predicted Compressor Head Coefficient at 80barA 


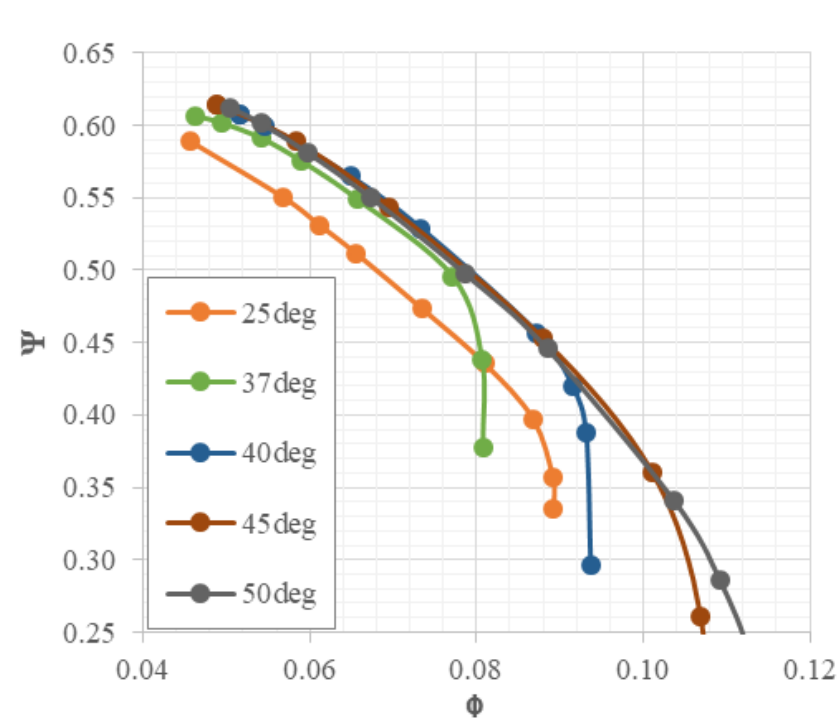

Figure 7: Predicted Compressor Head Coefficient at 85barA

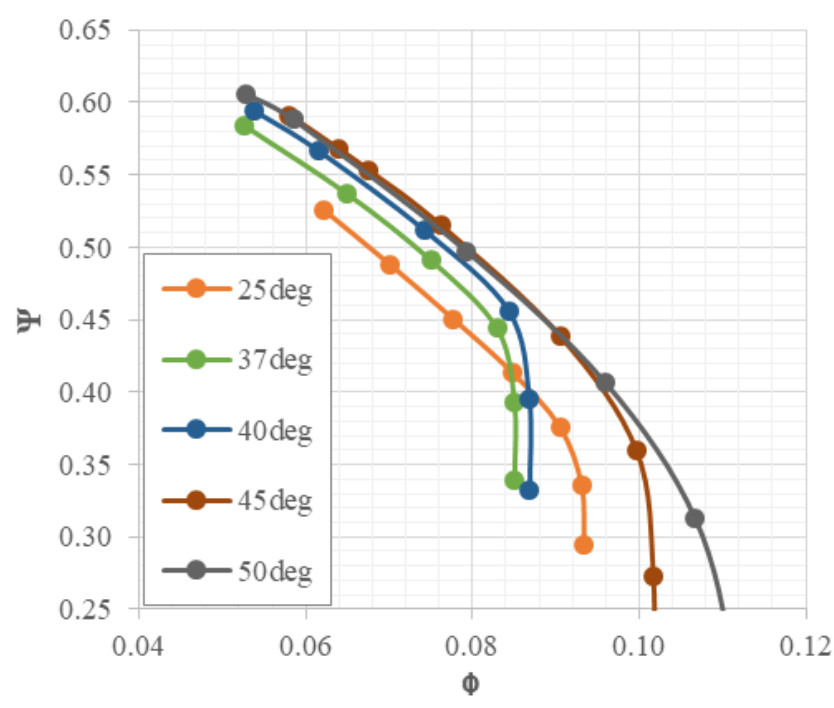
Figure 8: Predicted Compressor Head Coefficient at
90barA

Choke is the maximum flow that the impeller can pass at the given conditions and is evident on the right side of the performance map as characterized by a sharp reduction in head coefficient. Choke flow occurs when the flow at a point within the flowpath, typically the impeller, reaches a relative Mach number of 1.0. In this application, the choke flow rate is the greatest at high temperature and falls rapidly as the temperature approaches the critical temperature. At temperatures below the critical temperature the choke flow rate increases again.

The variation in choke flow can be expressed in terms of the choke margin, $C M$. The choke margin represents the fraction of flow above the design flow that the stage can operate to before reaching the choke limit. $C M$ is defined as:

$$
C M=\left(Q_{\text {choke }}-Q_{\text {design }}\right) / Q_{\text {design }}
$$

The change in choke flow is summarized as Figure 9 below, summarizes the variation in choke margin that is predicted for the various inlet conditions. It can be seen that the choke margin is very sensitive to inlet temperature when operating near the critical point. At the design point the choke margin is calculated to be $26 \%$, and falling to a minimum of less than $24 \%$ at an inlet temperature $5^{\circ} \mathrm{C}$ above the critical temperature. As the inlet pressure is reduced closer to the critical pressure the reduction in choke margin becomes more extreme. In fact, for inlet pressures below 80barA, this compressor is expected to be choked at its design point at certain inlet temperatures. This potential choked condition will only occur over a very narrow band in inlet temperature.

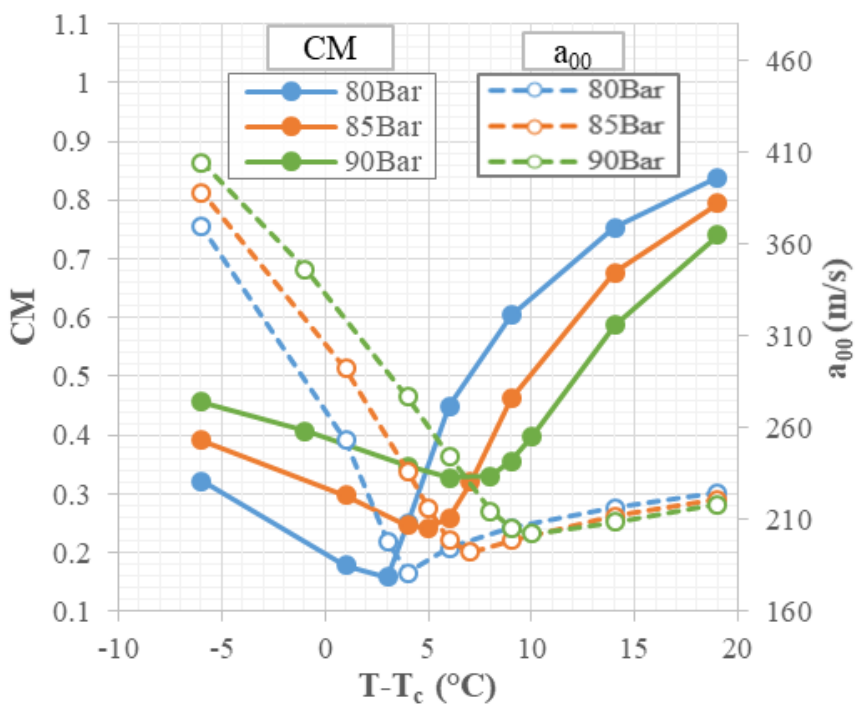

\section{Figure 9: Relation of Speed of Sound with Choke Margin}

The variation in the speed of sound of the fluid should be a consideration when selecting the design point of a compressor for a $\mathrm{sCO} 2$ application. In the case of the baseline compressor used for this study the compressor was sized near the point with the lowest sonic velocity. This will correspond to the highest inlet Mach number and be the most challenging design point. If a hotter or colder design point was chosen, than the compressor blading would be optimized to match a lower Mach number flow and may result in a design that could be prone to choking at off-design conditions. An understanding of the potential for choking should also be considered by the machine's control systems and it should be configured to prevent operating at conditions which may limit the compressor capacity and cycle performance.

The other noticeable change in the map is the variation in the magnitude of the head coefficient. Figure 10 shows how the head coefficient for each inlet pressure varies with temperature. The CFD results predict that the head will rise slightly as the inlet temperature falls to within $5^{\circ} \mathrm{C}$ above the critical temperature. As the temperature is further reduced the head coefficient begins to fall rapidly and asymptotes about $5 \%$ below the design performance. The variation in 
magnitude of the head coefficient on the design performance map of the compressor has been found to correlate well with the machine Mach number, $M_{U 2}$. As machine Mach number increases so does the head coefficient. A similar sensitivity of head coefficient to Mach number is often seen in centrifugal compressors.

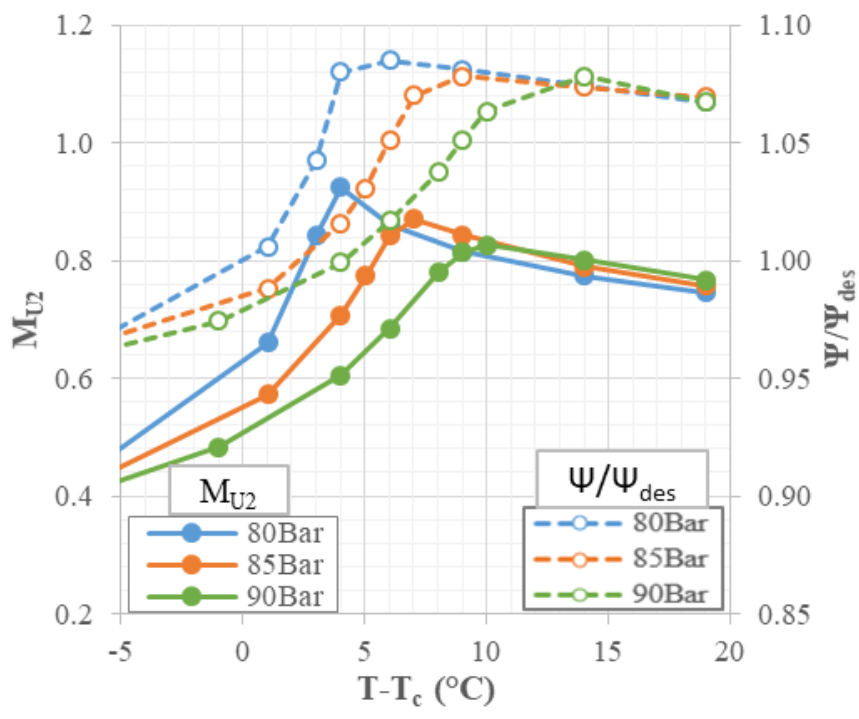

Figure 10: Variation in Design Point Head Coefficient Relative to the Critical Temperature

Figure 11, Figure 12 and Figure 13 show the variation in isentropic efficiency. The width of the map varies in proportion to the change in choke flow, as seen in the headflow plots. The magnitude of the efficiency also varies slightly as the inlet temperature changes.

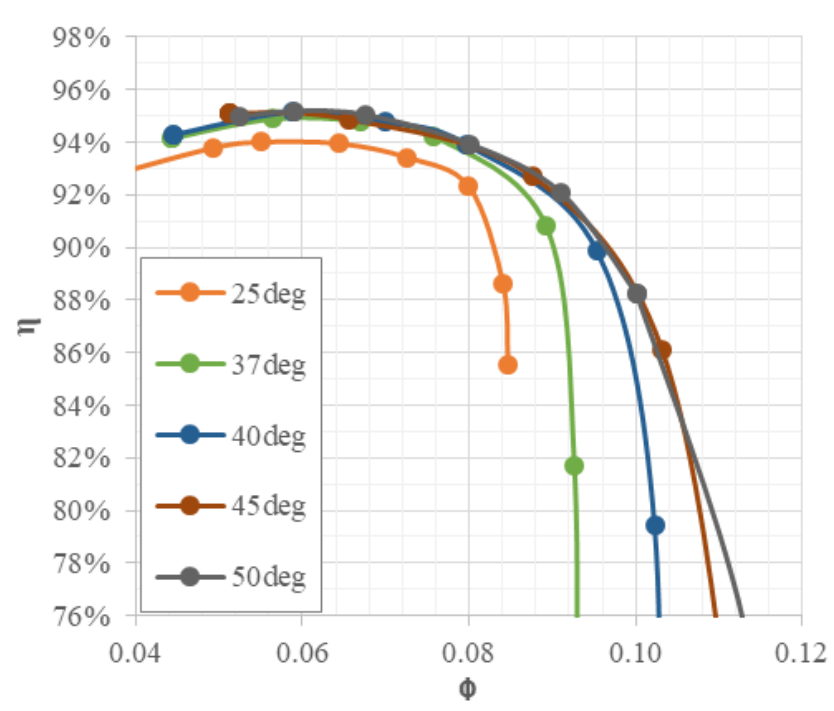

Figure 11: Predicted Compressor Isentropic Efficiency at 80barA

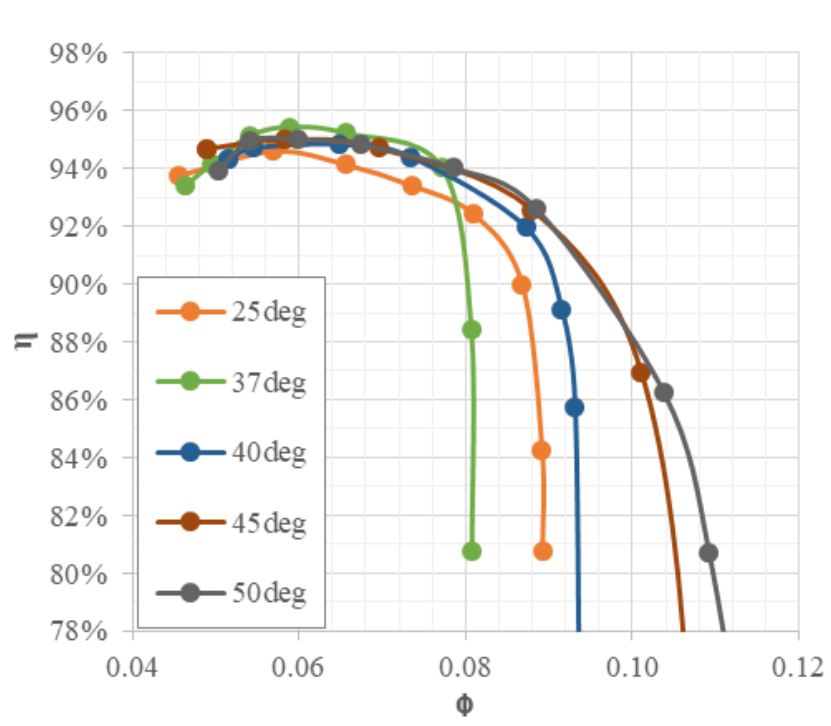

Figure 12: Predicted Compressor Isentropic Efficiency at 85barA

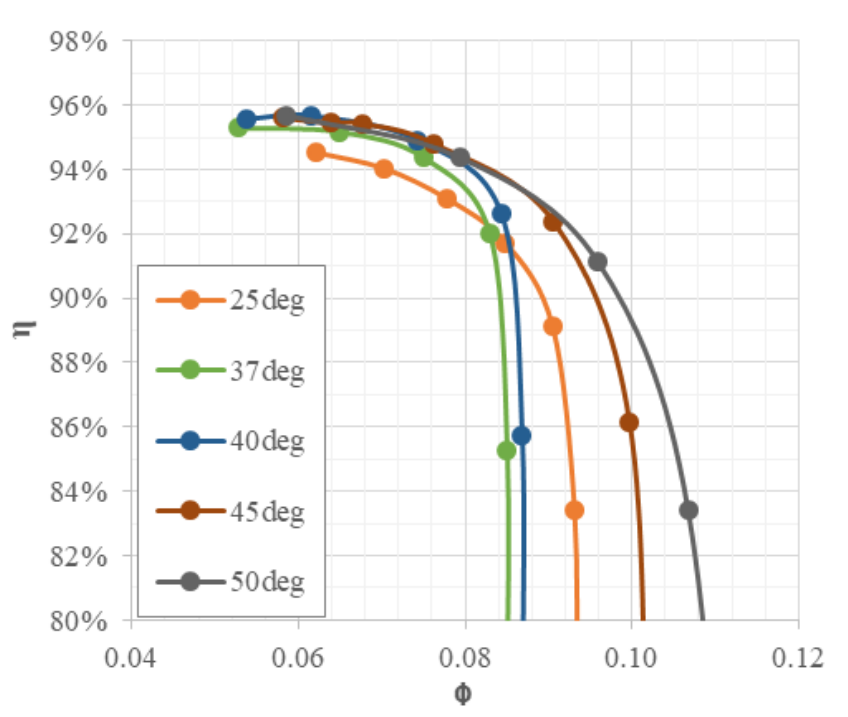

Figure 13: Predicted Compressor Isentropic Efficiency at 90barA

Figure 14 summarizes the trend in efficiency with variation in inlet conditions for the three different inlet pressures. At the design flow coefficient, the peak efficiency was predicted to change just 1.5 points as the inlet temperature was varied from 20 to $50^{\circ} \mathrm{C}$. At temperatures greater than $10^{\circ} \mathrm{C}$ above the critical temperature, the efficiency remains fairly constant with further increases in temperature. Below this point, a linear decrease in efficiency of approximately 1 point in efficiency for every $10^{\circ} \mathrm{C}$ was observed as the temperature was reduced. The decreasing trend in efficiency is likely due to a growing mismatch between the actual size of the impeller exducer and the optimal exducer area, which would increase as the compressibility falls and the change in density across the stage reduces at lower inlet temperatures.

The CFD analysis also showed a small spike in efficiency near the critical temperature. This spike may be a limitation of the table based fluid properties that are used in 
the CFD analysis to smoothly model the very rapid variation in properties that develop near the critical point. To more accurately capture the performance closest to the critical point, a multi-phase analysis would be most appropriate, but was beyond the scope of this work.

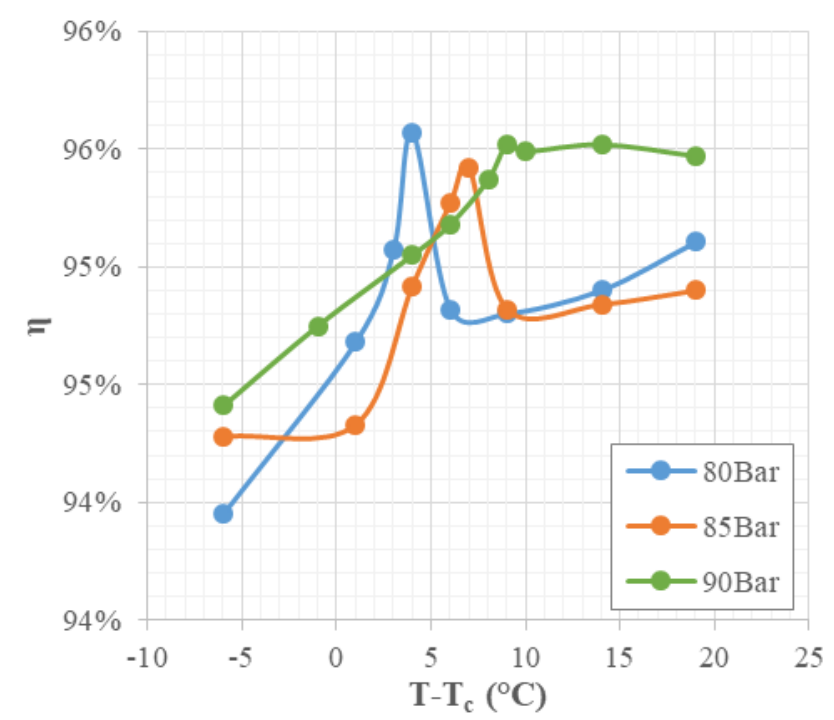

Figure 14: Predicted Trend in Compressor
Efficiency with Temperature

Although the efficiency falls slightly with temperature, an overall reduction in compression work still results as the fluid density increases. It is clearly beneficial from a cycle perspective to operate at lower compressor inlet temperatures despite some small potential loss in compression efficiency. The results of this study suggest that the stage can operate stably at low temperatures and with a minimal effect on performance.

\section{CONCLUSIONS}

For the main compressor in a sCO2 loop operating near the critical point, variations in fluid properties have been shown to have significant effect on the performance of the stage. Both the design and off-design performance of the compressor map will change as the stage is operated at alternate inlet conditions. Since the fluid is not an ideal gas, aerodynamic similarity laws cannot be used to accurately model the stage performance at off-design inlet conditions. Additionally, the fluid properties vary dramatically with small changes in the inlet temperature or pressure so offdesign cycle modelling must account for associated changes to the compressor performance in order to achieve realistic cycle performance estimates.

Although the design point map changes off-design, a CFD evaluation has shown that the performance variation can be approximated based on known changes to the operating conditions and a known baseline performance map. The main changes to the head coefficient include variability in the choke margin which has been shown to vary in proportion to the speed of sound of the gas at the inlet. The reduction in sonic velocity is so significant that some compressor designs may choke if their design point is selected too close to the critical point.
The magnitude of the head coefficient was also predicted to fall slightly at temperatures below the critical temperature. The magnitude of head coefficient is expected to vary directly proportional to changes in the machine Mach number. The effect of off-design inlet conditions on peak efficiency is modest. The peak efficiency is expected to remains nearly constant at temperatures above the critical temperature and falls gradually as the temperature is further reduced.

Based on these understandings of the key mechanisms controlling the characteristic of the compressor performance map a better approximation of off-design cycle performance is possible. The improved understanding of the off-design compressor performance will help in developing safe and reliable controls strategies and in making realistic cycle performance estimates.

\section{NOMENCLATURE}

$a_{00}=$ Speed of Sound at Inlet

$C M=$ Choke Margin $=\frac{\varphi_{\text {choke }}-\varphi_{\text {design }}}{\varphi_{\text {design }}}$

Cp = Specific Heat

$h=$ Enthalpy

$M=$ Mach Number

$M_{U 2}=U_{2} / a_{00}$

$Q=$ Volume Flow Rate

$U=$ Impeller Tip Speed

$\eta=\Delta h_{\text {isen }} / \Delta h$

$\phi=4 Q / \pi U_{2} d_{2}^{2}$

$\psi=2 \Delta h_{\text {isen }} / U_{2}^{2}$

\section{Subscripts}

2=Impeller Exit

$s=$ isentropic

\section{ACKNOWLEDGMENTS}

This material is based upon work supported by the Department of Energy, Office of Energy Efficiency and Renewable Energy (EERE), under Award Number DE0007114.

\section{DISCLAIMER}

This report was prepared as an account of work sponsored by an agency of the United States Government. Neither the United States Government nor any agency thereof, nor any of their employees, makes any warranty, express or implied, or assumes any legal liability or responsibility for the accuracy, completeness, or usefulness of any information, apparatus, product, or process disclosed, or represents that its use would not infringe privately owned rights. Reference herein to any specific commercial product, process, or service by trade name, trademark, manufacturer, or otherwise does not necessarily constitute or imply its endorsement, recommendation, or favoring by the United States Government or any agency thereof. The views and opinions of authors expressed herein do not necessarily state or reflect those of the United States Government or any agency thereof. 


\section{REFERENCES}

Angelino, G., (1969), "Real Gas Effects in Carbon Dioxide Cycles", ASME Paper No. 69-GT-103.

ASME, (1997), PTC 10-1997, "Performance Test Code on Compressors and Exhausters".

Bennett, J., Wilkes, J., Allison, T., Pelton, R., and Wygant, K., (2017), "Cycle Modeling and Optimization of an Integrally Geared sCO2 Compander," Proc. of the ASME Turbo Expo, GT2107-63707.

Buckingham, E., (1914), "On Physically Similar Systems: Illustration of the Use of Dimensional Equations, Phys. Rev., 4(4).

Clementoni, E., and Cox, T., (2018), "Effect of Compressor Inlet Pressure on Cycle Performance for a Supercritical Carbon Dioxide Brayton Cycle." Proc. of the ASME Turbo Expo, GT2018-75182, Oslo, Norway.

Dufour, G., Carbonneau, X., Cazalbou, J., and Chassaing, P., (2006), "Practical Use of Similarity and Scaling Laws for Centrifugal Compressor Design," Proc. Of the ASME Turbo Expo, GT2006-91227, Barcelona, Spain.

Fomin, Y.D., Ryzhov, V.N., Tsiok, E.N., Brazhkin, V.V., and Trachenko, K., (2014), "Thermodynamics and Widom Lines in Supercritical Carbon Dioxide" ArXiv Preparation. ArXiv14116849.

Holste, J.C., Hall, K.R., Eubank, P.T., Esper, G., Watson, M.Q., Warowny, W., Bailey, D.W., Young, J.G., and Bellomy, M.T., (1987) "Experimental (p, Vm, T) for Pure CO2 between 220 and 450K" The Journal of Chemical Thermodynamics 19, 1233-1250.

Japikse, D., (1996), Centrifugal Compressor Design and Performance, Concepts ETI, Inc.

Lemmon, E.W., Huber, M.L., and McLinden, M.O., NIST Standard Reference Database 23: Reference Fluid Thermodynamic and Transport Properties-REFPROP, Version 9.1, National Institute of Standards and Technology, Standard Reference Data Program, Gaithersburg, 2013.

Li, H., (2008), "Thermodynamics Properties of CO2 Mixtures and Their Applications in Advanced Power Cycles with CO2 Capture Process" Royal Institute of Technology, Stockholm.

Li, F., Pelton, R., and Jung, S., (2018), "Mechanical Design of A 3D Printed, Semi-Shrouded Centrifugal Compressor Impeller for Supercritical CO2 Application," Proceedings of GPPS Forum 18, Zurich, Switzerland.

Pelton, R., and Jung, S., (2018), "Design of a WideRange Centrifugal Compressor Stage for Supercritical CO2 Power Cycles", Journal of Eng. For Gas Turbines and Power, Vol. 140

Wilkes, J., Allison, T., Schmitt, J., Bennett, J., Wygant, K., Pelton, R., and Bosen, W., (2016) "Application of an Integrally Geared Compander to an $\mathrm{SCO} 2$ Recompression Brayton Cycle," $5^{\text {th }}$ Intl. Supercritical CO2 Power Cycle Symposium, March 28-31, San Antonio, TX. 